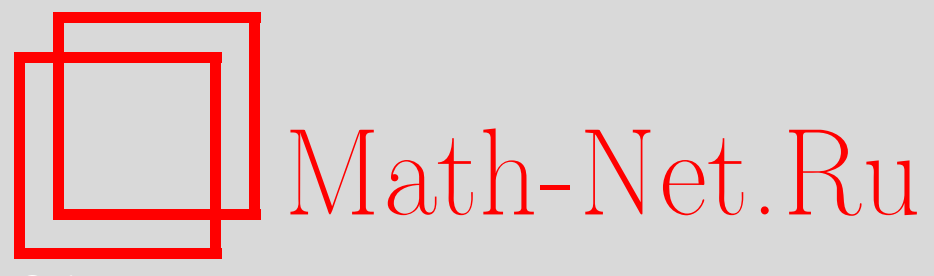

В. Ф. Бабенко, Н. В. Парфинович, Точные значения наилучших приближений классов периодических функций сплайнами дефекта 2, Матем. заметки, 2009, том 85, выпуск 4, 538-551

DOI: https://doi.org/10.4213/mzm4617

Использование Общероссийского математического портала Math-Net.Ru подразумевает, что вы прочитали и согласны с пользовательским соглашением http: //www . mathnet.ru/rus/agreement

Параметры загрузки:

IP: 44.207 .124 .84

26 апреля 2023 г., 18:16:19

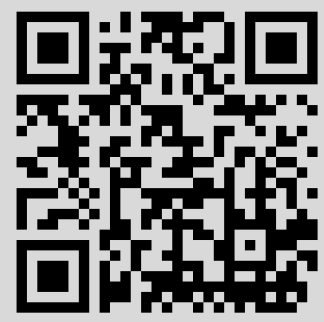


Том 85 выпуск 4 апрель 2009

УДК 517

\section{Точные значения наилучших приближений классов периодических функций сплайнами дефекта 2}

\section{В. Ф. Бабенко, Н. В. Парфинович}

Найдены точные значения наилучших $L_{1}$-приближений классов $W^{r} F, r \in \mathbb{N}$, периодических функций, $r$-я производная которых принадлежит заданному перестановочно-инвариантному множеству $F$, а также классов $W^{r} H^{\omega}$ периодических функций, $r$-я производная которых имеет заданную выпуклую вверх мажоранту $\omega(t)$ модуля непрерывности, подпространствами полиномиальных сплайнов порядка $m \geqslant r+1$ дефекта 2 с узлами в точках $2 k \pi / n, n \in \mathbb{N}, k \in \mathbb{Z}$. Показано, что эти подпространства являются экстремальными для поперечников по Колмогорову соответствующих функциональных классов.

Библиография: 28 названий.

1. Необходимые обозначения и определения. Пусть $L_{p}, 1 \leqslant p \leqslant \infty$, пространства $2 \pi$-периодических функций $f: \mathbb{R} \rightarrow \mathbb{R}$ с соответствующими нормами $\|\cdot\|_{L_{p}}=\|\cdot\|_{p}$, и пусть $p^{\prime}=p /(p-1)$.

Пусть $H$ - подпространство пространства $L_{p}$ и $g \in L_{p^{\prime}}$. Если для всех $h \in H$

$$
\int_{0}^{2 \pi} g(x) h(x) d x=0,
$$

то будем писать $g \perp H$. Если $H$ - подпространство констант, то вместо $g \perp H$ будем использовать обозначение $g \perp 1$.

Пусть множество $F \subset L_{1}$ таково, что $\{f \in F: f \perp 1\} \neq \varnothing$. Для $r=1,2, \ldots$ обозначим через $W^{r} F$ класс функций $f \in L_{1}$, у которых $(r-1)$-я производная $f^{(r-1)}\left(f^{(0)}=f\right)$ локально абсолютно непрерывна и $f^{(r)} \in F$. Отметим, что если $F$ - единичный шар пространства $L_{p}$, то множество $W^{r} F=W_{p}^{r}$ - это стандартный соболевский класс функций, у которых $(r-1)$-я производная локально абсолютно непрерывна и $\left\|f^{(r)}\right\|_{p} \leqslant 1$.

Для неотрицательной функции $f \in L_{1}$ обозначим через $r(f, t)$ неубывающую перестановку (см. [1; с. 130]) сужения функции $f$ на промежуток $[0,2 \pi]$. Если $g-$ произвольная функция из $L_{1}$, то положим (см. [2; с. 99])

$$
\Pi(g, t):=r\left(g_{+}, t\right)-r\left(g_{-}, 2 \pi-t\right),
$$

где $g_{ \pm}:=\max \{ \pm g(t), 0\}$.

Множество $F \in L_{1}$ назовем П-инвариантным, если из того, что $f \in F$ и $(g)=$ $\Pi(f)$, следует, что $g \in F$.

Приведем некоторые примеры П-инвариантных множеств.

(C) В.Ф. БАБЕнко, Н. В. ПАРФинович, 2009 
ПримеР 1. П-инвариантен единичный шар в любом вложенном в $L_{1}$ симметричном пространстве $2 \pi$-периодических функций, в частности, в пространствах $L_{p}$, $1 \leqslant p \leqslant \infty$, Орлича [3], Лоренца [4] и Марцинкевича [5].

ПримеР 2. Пусть $\varphi$ - произвольная неотрицательная неубывающая функция, определенная на $[0,+\infty)$. Тогда множество

$$
F=F(\varphi)=\left\{f \in L_{1}: \int_{0}^{2 \pi} \varphi(|f(t)|) d t \leqslant 1\right\}
$$

П-инвариантно.

ПримеР 3. Пусть $f$ - фиксированная функция из $L_{1}, f \perp 1$. Тогда П-инвариантным является каждое из множеств

$$
\begin{gathered}
F_{f, \Pi}=\left\{g \in L_{1}: \Pi(g)=\Pi(f)\right\}, \\
F_{f, r}=\left\{g \in L_{1}: r(|g|, t)=r(|f|, t), t \in[0,2 \pi)\right\}, \\
\bar{F}_{f, r}=\left\{g \in L_{1}: r(|g|, t) \leqslant r(|f|, t), t \in[0,2 \pi)\right\} .
\end{gathered}
$$

Пусть $C^{r}, r=0,1, \ldots,-$ пространство $r$ раз непрерывно дифференцируемых (непрерывных при $r=0$ ) $2 \pi$-периодических функций, $\omega(t)$ - произвольный фиксированный модуль непрерывности (см., например, [6; §6.1]). Класс функций $f \in C^{r}$, у которых при всех $t$

$$
\left|f^{(r)}(t)-f^{(r)}(t+\delta)\right| \leqslant \omega(\delta), \quad \delta \geqslant 0,
$$

будем обозначать через $W^{r} H^{\omega}$.

Наилучшее приближение класса $M \subset L_{p}$ множеством $H$ из $L_{p}$ в метрике $L_{p}-$ это величина

$$
E(M, H)_{p}=\sup _{f \in M} \inf _{h \in H}\|f-h\|_{p}
$$

Величины

$$
d_{n}\left(M, L_{p}\right)=\inf _{H_{n}} E(M, H)_{p},
$$

где $\inf _{H_{n}}$ берется по всем подпространствам $H_{n}$ пространства $L_{p}$, размерность которых не превосходит $n$, называются поперечниками Колмогорова класса $M$ в пространстве $L_{p}$. Подпространства $H_{n}$, которые реализуют точную нижнюю грань в правой части (1.1), называются экстремальными.

Для каждого натурального $n$ и $m=0,1, \ldots$ через $T_{2 n-1}$ обозначим пространство тригонометрических полиномов порядка не выше $n-1$, через $S_{2 n, m}^{1}$ - пространство $2 \pi$-периодических полиномиальных сплайнов порядка $m$ дефекта 1 с узлами в точках $j \pi / n, j \in \mathbb{Z}$, а через $S_{2 n, m}^{2}, n, m \in \mathbb{N}$ - пространство $2 \pi$-периодических полиномиальных сплайнов порядка $m$ дефекта 2 , с узлами в точках $t_{j}=2 j \pi / n$, $j \in \mathbb{Z}$.

Пусть $\varphi_{\lambda, m}(t), m \in \mathbb{N}, \lambda>0,-m$-й $(2 \pi / \lambda)$-периодический интеграл с нулевым средним значением на периоде от функции $\varphi_{\lambda, 0}(t)=\operatorname{sign} \sin \lambda t$.

При фиксированных $\omega(\cdot)$ и $n \in \mathbb{N}$ положим

$$
f_{n, 0}(\omega ; t)= \begin{cases}-\frac{1}{2} \omega\left(\frac{\pi}{n}-2 t\right), & 0 \leqslant t \leqslant \frac{\pi}{2 n}, \\ \frac{1}{2} \omega\left(2 t-\frac{\pi}{n}\right), & \frac{\pi}{2 n} \leqslant t \leqslant \frac{\pi}{n} .\end{cases}
$$


Через $f_{n, r}(\omega ; t), r=1,2, \ldots$, обозначим $r$-й $(2 \pi / n)$-периодический интеграл от функции $f_{n, 0}(\omega ; t)$ с нулевым средним значением на периоде.

2. Некоторые известные результаты. Хорошо известно (см., например, [6; теоремы $4.2 .4,5.4 .8,8.1 .13]$ ), что при всех $n, r=1,2, \ldots, 1 \leqslant p \leqslant \infty$ выполняются равенства

$$
\begin{aligned}
E\left(W_{p}^{r}, T_{2 n-1}\right)_{1} & =\left\|\varphi_{n, r}\right\|_{p^{\prime}}, \\
E\left(W_{p}^{r}, S_{2 n, m}^{1}\right)_{1} & =\left\|\varphi_{n, r}\right\|_{p^{\prime}}, \quad m=r-1, r, \ldots, \\
d_{2 n-1}\left(W_{p}^{r}, L_{1}\right)=d_{2 n}\left(W_{p}^{r}, L_{1}\right) & =\left\|\varphi_{n, r}\right\|_{p^{\prime}} .
\end{aligned}
$$

При этом пространства $T_{2 n-1}$ являются экстремальными пространствами для поперечников $d_{2 n-1}\left(W_{p}^{r}, L_{1}\right)$ и $d_{2 n}\left(W_{p}^{r}, L_{1}\right)$, а пространства $S_{2 n, m}^{1}$ при всех $m \geqslant r-1-$ для поперечников $d_{2 n}\left(W_{p}^{r}, L_{1}\right)$.

Равенство (2.1) при $p=1$ получено Никольским [7], для $p>1$ его установил Тайков [8], при $p=\infty$ независимо и другим методом этот результат получила Туровец [9]. Соотношение (2.2) установлено Лигуном [10]. Оценку снизу для нечетных поперечников при $p=1$ получили независимо Маковоз [11] и Субботин [12], [13], а при $p=\infty$ - Маковоз [14]. Оценка снизу для четных поперечников при $p=1, \infty$ принадлежит Рубану (см., например [1; гл. 10]). Для $1<p<\infty$ соотношение (2.3) независимо и разными методами получили Лигун [15], Маковоз [16], Пинкус [17].

В [18] установлено, что для произвольного П-инвариантного множества $F$ и любых $n, r=1,2, \ldots, m \geqslant r$ имеют место следующие равенства

$$
\begin{gathered}
E\left(W^{r} F, T_{2 n-1}\right)_{1}=E\left(W^{r} F, S_{2 n, m}^{1}\right)_{1}=\sup _{\substack{f \in F \\
f \perp 1}} \int_{0}^{2 \pi} \Pi(f, t) \Pi\left(\varphi_{n, r}, t\right) d t, \\
d_{2 n-1}\left(W^{r} F, L_{1}\right)=d_{2 n}\left(W^{r} F, L_{1}\right)=\sup _{\substack{f \in F \\
f \perp 1}} \int_{0}^{2 \pi} \Pi(f, t) \Pi\left(\varphi_{n, r}, t\right) d t .
\end{gathered}
$$

При этом пространства $T_{2 n-1}$ являются экстремальными пространствами для поперечников $d_{2 n-1}\left(W^{r} F, L_{1}\right)$ и $d_{2 n}\left(W^{r} F, L_{1}\right)$, а пространства $S_{2 n, m}^{1}$ при всех $m \geqslant r-$ для поперечников $d_{2 n}\left(W^{r} F, L_{1}\right)$.

Корнейчук [19]-[21] (см. также [6; теоремы 7.2.2, 7.2.5]) доказал, что для любого выпуклого вверх модуля непрерывности $\omega(t)$ при всех $n=1,2, \ldots, r=0,1, \ldots$, $m \geqslant r$ имеет место соотношение

$$
E\left(W^{r} H^{\omega}, T_{2 n-1}\right)_{1}=E\left(W^{r} H^{\omega}, S_{2 n, m}^{1}\right)_{1}=\left\|f_{n, r}(\omega ; \cdot)\right\|_{1} .
$$

Известно также, что

$$
d_{2 n-1}\left(W^{r} H^{\omega}, L_{1}\right)=d_{2 n}\left(W^{r} H^{\omega}, L_{1}\right)=\left\|f_{n, r}(\omega ; \cdot)\right\|_{1},
$$

причем поперечники $d_{2 n-1}\left(W^{r} H^{\omega}, L_{1}\right)$ и $d_{2 n}\left(W^{r} H^{\omega}, L_{1}\right)$ реализует подпространство $T_{2 n-1}$, а поперечник $d_{2 n}\left(W^{r} H^{\omega}, L_{1}\right)$ - подпространство $S_{2 n, m}^{1}, m \geqslant r$. Оценки снизу для нечетных поперечников получены Моторным и Рубаном [22], а для четных Рубаном [23].

3. Аппроксимация сплайнами дефекта 2. Формулировка результатов. Напомним, что $S_{2 n, m}^{2}, n, m \in \mathbb{N},-$ линейное пространство $2 \pi$-периодических полиномиальных сплайнов порядка $m$ дефекта 2 с узлами в точках $t_{j}=2 j \pi / n, j \in \mathbb{Z}$. 
Отметим, что размерность этого пространства при любом $m$ равна $2 n$ (см., например, [6; следствие 2.3.7]).

В данной работе найдены точные значения наилучших $L_{1}$-приближений классов $W^{r} F$ и $W^{r} H^{\omega}$ сплайнами из $S_{2 n, m}^{2}$ при $m \geqslant r$ и $m \geqslant r+1$ соответственно. Справедливы следующие теоремы.

Теорема 1. Пусть $n, r=1,2, \ldots, m=r, r+1, \ldots, 1 \leqslant p \leqslant \infty, F-$ произвольное П-инвариантное множество $2 \pi$-периодических функций. Тогда

$$
E\left(W^{r} F, S_{2 n, m}^{2}\right)_{1}=\sup _{\substack{f \in F \\ f \perp 1}} \int_{0}^{2 \pi} \Pi(f, t) \Pi\left(\varphi_{n, r}, t\right) d t .
$$

В частности,

$$
E\left(W_{p}^{r}, S_{2 n, m}^{2}\right)_{1}=\left\|\varphi_{n, r}\right\|_{p^{\prime}}
$$

Tеорема 2. Пусть $n, r=1,2, \ldots, m=r+1, r+2, \ldots, \omega(t)$ - выпуклый вверх модуль непрерывности. Тогда

$$
E\left(W^{r} H^{\omega}, S_{2 n, m}^{2}\right)_{1}=\left\|f_{n, r}(\omega, \cdot)\right\|_{1} .
$$

Результаты теорем 1 и 2 вместе с соотношениями (2.4) и (2.6) показывают, что $S_{2 n, m}^{2}$ наряду с $T_{2 n-1}$ и $S_{2 n, m}^{1}$ являются экстремальными подпространствами для поперечников $d_{2 n}\left(W^{r} F, L_{1}\right)$ (при $m \geqslant r$ ) и $d_{2 n}\left(W^{r} H^{\omega}, L_{1}\right)($ при $m \geqslant r+1$ ).

Существенную роль при доказательстве теорем 1 и 2 будет играть следующая лемма, проясняющая смысл ортогональности функции $g$ подпространству $S_{2 n, m}^{2}$.

ЛЕмма 1. Для того чтобъ $2 \pi$-периодическая функиия $f \in L_{1}$ была ортогональна пространству $S_{2 n, m}^{2}, n, m \in \mathbb{N}$, необходимо и достаточно, чтобъ для $(m+1)$-й $2 \pi$-периодической первообразной $f_{m+1}(t)$ функции $f(t)$

$f_{m+1}\left(t_{1}\right)=f_{m+1}\left(t_{2}\right)=\cdots=f_{m+1}\left(t_{n}\right), \quad f_{m+1}^{\prime}\left(t_{1}\right)=f_{m+1}^{\prime}\left(t_{2}\right)=\cdots=f_{m+1}^{\prime}\left(t_{n}\right)=0$.

Кроме того, при доказательстве теорем 1 и 2 нами существенно будет использована следующая теорема, которая представляет и самостоятельный интерес.

Tеорема 3. Пусть $n, l \in \mathbb{N}, l \geqslant 2, t_{j}=2 j \pi / n$ для $j=1,2, \ldots, n$. Пусть $\widetilde{\varphi}(t)=$ $\varphi_{n, l}(t+\alpha)+\left\|\varphi_{n, l}\right\|_{\infty}$, где $\alpha$ выбрано из условия $\widetilde{\varphi}(0)=0$. Тогда для любой функи,и $g \in W_{\infty}^{l}$ maкой, что

$$
g\left(t_{1}\right)=g\left(t_{2}\right)=\cdots=g\left(t_{n}\right), \quad g^{\prime}\left(t_{1}\right)=g^{\prime}\left(t_{2}\right)=\cdots=g^{\prime}\left(t_{n}\right)=0,
$$

имеют место утверждения

а) для любого $t \in \mathbb{R}|g(t)-g(0)| \leqslant \widetilde{\varphi}(t)$, причем, если $|g(t)-g(0)|$ отлична от $\widetilde{\varphi}(t)$, то знак равенства имеет место только в точках $t_{j}, j=1,2, \ldots, n$;

b) $\left\|g^{(k)}\right\|_{\infty} \leqslant\left\|\varphi_{n, l-k}\right\|_{\infty}, k=1,2, \ldots, l$.

4. Доказательства. Сначала мы докажем лемму 1, затем теорему 3 , а уже затем теоремы 1 и 2.

ДОКАЗАТЕЛЬСТво ЛЕмМЫ 1. Известно [6; следствие 2.3.6], что любой сплайн $s$ из $S_{2 n, m}^{2}$ единственным образом представим в виде

$$
s(t)=a_{0}+\sum_{j=1}^{n} a_{j} B_{m+1}\left(t-t_{j}\right)+\sum_{j=1}^{n} b_{j} B_{m}\left(t-t_{j}\right),
$$


где

$$
\begin{gathered}
a_{0}=\frac{1}{2 \pi} \int_{0}^{2 \pi} s(t) d t, \quad \sum_{j=1}^{n} a_{j}=0, \\
B_{m}(t)=\sum_{k=1}^{n} \frac{\cos (k t-\pi m / 2)}{k^{m}}, \quad m=1,2, \ldots,
\end{gathered}
$$

- ядро Бернулли.

Пусть $f \perp S_{2 n, m}^{2}$, т.е.

$$
\int_{0}^{2 \pi} f(t) s(t) d t=0 \quad \text { для любого } \quad s \in S_{2 n, m}^{2} .
$$

Прежде всего заметим, что так как $S_{2 n, m}^{2}$ содержит константы, то $f \perp 1$. С учетом (4.1) получим

$$
\begin{aligned}
\int_{0}^{2 \pi} & f(t)\left(a_{0}+\sum_{j=1}^{n} a_{j} B_{m+1}\left(t-t_{j}\right)+\sum_{j=1}^{n} b_{j} B_{m}\left(t-t_{j}\right)\right) d t \\
& =\sum_{j=1}^{n} a_{j} \int_{0}^{2 \pi} f(t) B_{m+1}\left(t-t_{j}\right) d t+\sum_{j=1}^{n} b_{j} \int_{0}^{2 \pi} f(t) B_{m}\left(t-t_{j}\right) d t \\
& =\sum_{j=1}^{n} a_{j} f_{m+1}\left(t_{j}\right)+\sum_{j=1}^{n} b_{j} f_{m+1}^{\prime}\left(t_{j}\right)=0 .
\end{aligned}
$$

Пусть $a_{1}=a_{2}=\cdots=a_{n}=0$, тогда для выполнения последнего равенства необходимо, чтобы для любого набора $b=\left(b_{1}, b_{2}, \ldots, b_{n}\right)$ было $\sum_{j=1}^{n} b_{j} f_{m+1}^{\prime}\left(t_{j}\right)=0$, а это равенство возможно, только если $f_{m+1}^{\prime}\left(t_{j}\right)=0$ для всех $j=1,2, \ldots, n$.

Пусть теперь $b_{1}=b_{2}=\cdots=b_{n}=0$. Равенство $\sum_{j=1}^{n} a_{j} f_{m+1}\left(t_{j}\right)=0$ равносильно ортогональности векторов $\bar{a}=\left(a_{1}, a_{2}, \ldots, a_{n}\right)$ и $\bar{f}_{m+1}=\left(f_{m+1}\left(t_{1}\right), f_{m+1}\left(t_{2}\right), \ldots\right.$, $\left.f_{m+1}\left(t_{n}\right)\right)$. Условие $\sum_{j=1}^{n} a_{j}=0$ выделяет в пространстве $\mathbb{R}^{n}$ гиперплоскость с нормальным вектором $(1,1, \ldots, 1)$, который в свою очередь параллелен вектору $\bar{f}_{m+1}$, а следовательно, $f_{m+1}\left(t_{1}\right)=f_{m+1}\left(t_{2}\right)=\cdots=f_{m+1}\left(t_{n}\right)$. Необходимость установлена. В силу соотношения (4.2) и условия $\sum_{j=1}^{n} a_{j}=0$ достаточность очевидна.

Лемма 1 доказана.

ДОКАЗАТЕЛЬСТво ТЕОРЕМЫ 3 . Пусть $\widetilde{g}(t)=g(t)-g(0)$. Функции $\widetilde{\varphi}(t)$ и $\widetilde{g}(t)$ имеют нули кратности 2 в точках $t_{j}, j \in \mathbb{Z}$.

Для доказательства утверждения а) предположим, что найдется $t_{*}$ такое, что $\left|\widetilde{g}\left(t_{*}\right)\right|>\widetilde{\varphi}\left(t_{*}\right)$. При подходящем $0<|\lambda|<1$ получим $\lambda \widetilde{g}\left(t_{*}\right)=\widetilde{\varphi}\left(t_{*}\right)$. Пусть $\delta(t)=$ $\widetilde{\varphi}(t)-\lambda \widetilde{g}(t)$. Так как $\left\|\lambda \widetilde{g}^{(l)}(t)\right\|_{\infty}<1$, а $\left\|\widetilde{\varphi}^{(l)}(t)\right\|_{\infty}=1$, то ни на одном отрезке $[\alpha, \beta]$ положительной длины функция $\delta^{(l)}(t)$, а значит, и $\delta(t)$ не обращаются тождественно в нуль, так что все нули $\delta(t)$ являются изолированными. Ясно, что функция $\delta(t)$ имеет нуль в точке $t_{*}$ и нули кратности 2 в точках $t_{j}, j \in \mathbb{Z}$, т.е. всего на периоде у функции $\delta(t)$ имеется по крайней мере $2 n+1$ нуль с учетом кратности. Тогда у $\delta^{\prime}(t)$ будет по крайней мере $2 n+1$ различных нулей, а у $\delta^{(l)}(t), l \geqslant 2$, будет не менее $2 n+2$ перемен знака на периоде. Однако $\delta^{(l)}(t)=\varphi_{n, 0}(t)-\lambda \widetilde{g}^{(l)}(t)$ имеет на периоде ровно $2 n$ перемен знака, и мы получили противоречие, которое доказывает утверждение а). 
Докажем утверждение b). Пусть $t_{\max } \in(0,2 \pi)$ таково, что $\left|\widetilde{g}^{\prime}\left(t_{\max }\right)\right|=\left\|\widetilde{g}^{\prime}\right\|_{\infty}$, и $j \in \mathbb{Z}$ выбрано из условия $t_{\max } \in\left(t_{j}, t_{j+1}\right)$. Предположив, что $\left|\widetilde{g}^{\prime}\left(t_{\max }\right)\right|>\left\|\widetilde{\varphi}^{\prime}\right\|_{\infty}$, мы сможем указать такое $0<|\lambda|<1$, что

$$
\lambda \widetilde{g}^{\prime}\left(t_{\max }\right)=\left\|\widetilde{\varphi}^{\prime}\right\|_{\infty}=\widetilde{\varphi}^{\prime}\left(t_{j}+\frac{\pi}{2 n}\right)=\left|\widetilde{\varphi}^{\prime}\left(t_{j}-\frac{\pi}{2 n}\right)\right| .
$$

Кроме того,

$$
\lambda \widetilde{g}^{\prime}\left(t_{j}\right)=\lambda \widetilde{g}^{\prime}\left(t_{j+1}\right)=\widetilde{\varphi}^{\prime}\left(t_{j}\right)=\widetilde{\varphi}^{\prime}\left(t_{j+1}\right)=0 .
$$

Ясно, что функции $\lambda \widetilde{g}^{\prime}(t)$ и $\widetilde{\varphi}^{\prime}(t)$ удовлетворяют условиям теоремы Колмогорова о сравнении производных [24] (см. также [6; теорема 3.3.2]). Учитывая это обстоятельство и соотношения (4.3), (4.4), нетрудно установить (достаточно, например, воспользоваться предложением 5.6.6 из [1]), что

$$
t_{\max } \in\left[t_{j}+\frac{\pi}{2 n}, t_{j+1}-\frac{\pi}{2 n}\right] .
$$

Ясно, что найдутся точки $t_{j}^{1} \in\left[t_{j}, t_{j}+\pi / n\right], t_{j}^{2} \in\left[t_{j}+\pi / n, t_{j+1}\right]$ такие, что

$$
\lambda \widetilde{g}^{\prime}\left(t_{j}^{1}\right)=\lambda \widetilde{g}^{\prime}\left(t_{j}^{2}\right)=0, \quad t_{\max } \in\left(t_{j}^{1}, t_{j}^{2}\right), \quad \lambda \widetilde{g}^{\prime}(t)>0, \quad t \in\left(t_{j}^{1}, t_{j}^{2}\right) .
$$

Снова используя предложение 5.6.6 из [1], заключаем, что

$$
t_{\max }-t_{j}^{1} \geqslant \frac{\pi}{2 n} \quad \text { и } \quad t_{j}^{2}-t_{\max } \geqslant \frac{\pi}{2 n}
$$

и, следовательно,

$$
t_{j}^{1}-t_{j}+t_{j+1}-t_{j}^{2} \leqslant \frac{\pi}{n}
$$

В силу предложения 5.6.5 из [1] с учетом того, что

$$
\lambda \widetilde{g}^{\prime}(t) \geqslant \widetilde{\varphi}^{\prime}\left(t+\frac{\pi}{2 n}-t_{\max }\right), \quad t \in\left(t_{\max }-\frac{\pi}{2 n}, t_{\max }+\frac{\pi}{2 n}\right),
$$

заключаем, что

$$
\int_{t_{j}^{1}}^{t_{j}^{2}} \lambda \widetilde{g}^{\prime}(t) d t \geqslant \int_{t_{\max }-\pi / 2 n}^{t_{\max }+\pi / 2 n} \widetilde{\varphi}^{\prime}\left(t+\frac{\pi}{2 n}-t_{\max }\right) d t=\int_{t_{j}}^{t_{j}+\pi / n} \widetilde{\varphi}^{\prime}(t) d t,
$$

и так как $\int_{t_{j}}^{t_{j+1}} \lambda \widetilde{g}^{\prime}(t) d t=0$, то

$$
\left|\int_{\left[t_{j}, t_{j+1}\right] \backslash\left(t_{j}^{1}, t_{j}^{2}\right)} \lambda \widetilde{g}^{\prime}(t) d t\right| \geqslant \int_{t_{j}}^{t_{j}+\pi / n} \widetilde{\varphi}^{\prime}(t) d t .
$$

С другой стороны (здесь мы снова используем (4.4) и предложение 5.6 .5 из [1]) для $t \in\left(t_{j}, t_{j}^{\prime}\right) \cup\left(t_{j}^{2}, t_{j+1}\right)$ получаем $\left|\lambda \widetilde{g}^{\prime}(t)\right| \leqslant\left|\widetilde{\varphi}^{\prime}(t)\right|$. Учитывая это неравенство и (4.6), заключаем, что

$$
\left|\int_{\left[t_{j}, t_{j+1}\right] \backslash\left(t_{j}^{1}, t_{j}^{2}\right)} \lambda \widetilde{g}^{\prime}(t) d t\right| \leqslant \int_{\left[t_{j}, t_{j+1}\right] \backslash\left(t_{j}^{1}, t_{j}^{2}\right)}\left|\widetilde{\varphi}^{\prime}(t)\right| d t \leqslant \int_{t_{j}}^{t_{j}+\pi / n} \widetilde{\varphi}^{\prime}(t) d t .
$$

Сопоставляя (4.9) и (4.10), видим, что

$$
\left|\int_{\left[t_{j}, t_{j+1}\right] \backslash\left(t_{j}^{1}, t_{j}^{2}\right)} \lambda \widetilde{g}^{\prime}(t) d t\right|=\left|\int_{t_{j}}^{t_{j}+\pi / n} \widetilde{\varphi}^{\prime}(t) d t\right|,
$$


но тогда и

$$
\int_{t_{j}^{1}}^{t_{j}^{2}} \lambda \widetilde{g}^{\prime}(t) d t=\int_{t_{j}}^{t_{j}+\pi / n} \widetilde{\varphi}^{\prime}(t) d t
$$

Вспоминая, что $\lambda \widetilde{g}^{\prime}(t) \geqslant \widetilde{\varphi}^{\prime}\left(t+\pi / 2 n-t_{\max }\right)$ для всех $t \in\left[t_{\max }-\pi / 2 n, t_{\max }+\pi / 2 n\right]$, видим, что равенство (4.11) может выполняться только в том случае, когда $\lambda \widetilde{g}^{\prime}(t)=$ $\widetilde{\varphi}^{\prime}\left(t+\pi / 2 n-t_{\max }\right)$ для всех $t \in\left[t_{\max }-\pi / 2 n, t_{\max }+\pi / 2 n\right]$, но это невозможно, так как $\left|\lambda \widetilde{g}^{(l)}(t)\right|<1$ для почти всех $t$.

Таким образом, нами доказана справедливость неравенства $\left\|g^{\prime}\right\|_{\infty} \leqslant\left\|\varphi_{n, l}\right\|_{\infty}$, откуда, используя теорему сравнения [6; теорема 3.3 .2$]$, получим неравенства

$$
\left\|g^{(k)}\right\|_{\infty} \leqslant\left\|\varphi_{n, l-k}\right\|_{\infty}, \quad k=1,2, \ldots, l .
$$

Теорема доказана.

ДоКАЗАТЕЛЬСТво тЕОРЕМЫ 1. Используя теорему двойственности Никольского [25] для наилучшего $L_{1}$-приближения подпространством (см. также [6; теорема 1.4.1]), получим

$$
E:=E\left(W^{r} F, S_{2 n, m}^{2}\right)_{1}=\sup _{f \in W^{r} F} \sup _{\substack{\|g\|_{\infty} \leqslant 1 \\ g \perp S_{2 n, m}^{2}}} \int_{0}^{2 \pi} f(t) g(t) d t .
$$

После $r$-кратного интегрирования по частям с использованием леммы 1 можем написать

$$
E=\sup _{\substack{f \in F \\ f \perp 1}} \sup _{\substack{g \in W_{\infty}^{r} \\ g^{(r)} \perp S_{2 n, m}^{2}}} \int_{0}^{2 \pi} f(t) g(t) d t=\sup _{\substack{g \in W_{\infty}^{m+1} \\ g\left(t_{1}\right)=\cdots=g\left(t_{n}\right) \\ g^{\prime}\left(t_{1}\right)=\cdots=g^{\prime}\left(t_{n}\right)=0}} \sup _{\substack{f \in F \\ f \perp 1}} \int_{0}^{2 \pi} f(t) g^{(m-r+1)}(t) d t,
$$

где $t_{j}=2 j \pi / n, j=1,2, \ldots, n$.

Учитывая предложение 1.3.4 из [26], окончательно получаем

$$
E=\sup _{\substack{f \in F \\ f \perp 1}} \sup _{\substack{g \in W_{\infty}^{r} \\ g^{(r)} \perp S_{2 n, m}^{2}}} \int_{0}^{2 \pi} f(t) g(t) d t=\sup _{\substack{g \in W_{\infty}^{m+1} \\ g\left(t_{1}\right)=\cdots=g\left(t_{n}\right) \\ g^{\prime}\left(t_{1}\right)=\cdots=g^{\prime}\left(t_{n}\right)=0}} \sup _{\substack{f \in F \\ f \perp 1}} \int_{0}^{2 \pi} \Pi(f, t) \Pi\left(g^{(m-r+1)}, t\right) d t .
$$

Покажем, что для любой функции $g \in W_{\infty}^{m+1}$ такой, что $g\left(t_{1}\right)=\cdots=g\left(t_{n}\right)$ и $g^{\prime}\left(t_{1}\right)=\cdots=g^{\prime}\left(t_{n}\right)=0$, имеет место неравенство

$$
\int_{0}^{t} r\left(\left(g^{(m-r+1)}\right)_{ \pm}, u\right) d u \leqslant \int_{0}^{t} r\left(\left(\varphi_{n, r}\right)_{ \pm}, u\right) d u, \quad 0 \leqslant t \leqslant 2 \pi .
$$

Если $m>r$, то используя утверждение b) теоремы 3 при $l=m+1$, получим $\left\|g^{(m-r)}\right\|_{\infty} \leqslant\left\|\varphi_{n, r+1}\right\|_{\infty}$. Таким образом, выполняются условия теоремы 3.3 .4 из [6], в силу которой неравенство (4.13) имеет место.

В случае $m=r$ сравним интегралы

$$
\int_{0}^{t} r\left(g_{ \pm}^{\prime}, u\right) d u \quad \text { и } \quad \int_{0}^{t} r\left(\left(\varphi_{n, r}\right)_{ \pm}, u\right) d u, \quad t \in[0,2 \pi],
$$

непосредственно. 
Пусть $\widetilde{\varphi}(t)=\varphi_{n, r+1}(t+\mu)+\left\|\varphi_{n, r+1}\right\|_{\infty}$, где $\mu$ выбрано из условия $\widetilde{\varphi}(0)=0$. Отметим, что $r\left(\left(\widetilde{\varphi}^{\prime}\right)_{+}, t\right)=r\left(\left(\varphi_{n, r}\right)_{+}, t\right)$ для всех $t \in[0,2 \pi]$.

Обозначим через $g_{j}^{\prime}$ и $\widetilde{\varphi}_{j}^{\prime}$ сужения на $\left[t_{j}, t_{j+1}\right], j=0,1, \ldots, n-1$, функций $g^{\prime}$ и $\widetilde{\varphi}^{\prime}$ соответственно.

Сначала докажем, что для всех $j=0,1, \ldots, n-1$ имеет место неравенство

$$
\left.\int_{0}^{t} r\left(\left(g_{j}^{\prime}\right)_{ \pm}, u\right) d u \leqslant \int_{0}^{t} r\left(\widetilde{\varphi}_{j}^{\prime}\right)_{ \pm}, u\right) d u, \quad t \in\left[0, \frac{2 \pi}{n}\right] .
$$

В первую очередь отметим, что для всех $j=0,1, \ldots, n-1$ имеет место соотношение

$$
\int_{t_{j}}^{t_{j+1}}\left(g_{j}^{\prime}\right)_{ \pm}(t) d t \leqslant \int_{t_{j}}^{t_{j+1}}\left(\widetilde{\varphi}_{j}^{\prime}\right)_{ \pm}(t) d t
$$

Действительно, в силу условия $\int_{t_{j}}^{t_{j+1}} g_{j}^{\prime}(t) d t=0$ видим, что $\operatorname{supp}\left(\left(g_{j}^{\prime}\right)_{ \pm}\right) \neq \varnothing$, если $g_{j}^{\prime}$ - не тождественный нуль на $\left[t_{j}, t_{j+1}\right]$. Пусть, для определенности,

$\left.\operatorname{mes} \operatorname{supp}\left(\left(g_{j}^{\prime}\right)_{+}\right) \geqslant \operatorname{mes} \operatorname{supp}\left(\widetilde{\varphi}_{j}^{\prime}\right)_{ \pm}\right) \quad$ и $\left.\quad \operatorname{mes} \operatorname{supp}\left(\left(g_{j}^{\prime}\right)_{-}\right) \leqslant \operatorname{mes} \operatorname{supp}\left(\widetilde{\varphi}_{j}^{\prime}\right)_{ \pm}\right)$.

Используя предложение 5.6.5 из [1], нетрудно убедиться в том, что

$$
\int_{t_{j}}^{t_{j+1}}\left(g_{j}^{\prime}\right)_{-}(t) d t \leqslant \int_{t_{j}}^{t_{j+1}}\left(\widetilde{\varphi}_{j}^{\prime}\right)_{ \pm}(t) d t
$$

и, более того, что справедливо неравенство (4.14) для отрицательных частей. Учитывая (4.16) и тот факт, что

$$
\int_{t_{j}}^{t_{j+1}}\left(g_{j}^{\prime}\right)_{+}(t) d t=\int_{t_{j}}^{t_{j+1}}\left(g_{j}^{\prime}\right)_{-}(t) d t
$$

получим (4.15). Докажем, что (4.14) имеет место и для положительных частей, т.е.

$$
\left.\int_{0}^{t} r\left(\left(g_{j}^{\prime}\right)_{+}, u\right) d u \leqslant \int_{0}^{t} r\left(\widetilde{\varphi}_{j}^{\prime}\right)_{+}, u\right) d u, \quad t \in\left[0, \frac{2 \pi}{n}\right],
$$

для каждого $j=0,1, \ldots, n-1$. При этом будем следовать схеме доказательства теоремы 6.8.1 из [1].

Положим

$$
\delta(t)=r\left(\left(\widetilde{\varphi}_{j}^{\prime}\right)_{+}, t\right)-r\left(\left(g_{j}^{\prime}\right)_{+}, t\right), \quad t \in\left[0, \frac{2 \pi}{n}\right] .
$$

Если $\delta(t) \geqslant 0$ для всех $t \in[0,2 \pi / n]$, то справедливость (4.17) очевидна. Заметим, что когда $\delta(t)$ меняет знак на промежутке $(0,2 \pi / n)$, справедливость $(4.17)$ достаточно установить лишь во всех точках промежутка $(0,2 \pi / n)$, являющихся нулями $\delta(t)$ и правыми концами интервалов, на которых $\delta(t)<0$.

Пусть $\delta(\xi)=0$ для некоторого $\xi \in(0,2 \pi / n)$ и найдется $\varepsilon>0$ такое, что $\delta(t)<0$ для $0<\xi-\varepsilon<t<\xi$.

Положим

$$
z=r\left(\left(g_{j}^{\prime}\right)_{+}, \xi\right)=r\left(\left(\widetilde{\varphi}_{j}^{\prime}\right)_{+}, \xi\right)
$$

Тогда

$$
\int_{0}^{\xi} r\left(\left(g_{j}^{\prime}\right)_{+}, t\right) d t=\int_{e_{j}(z)}\left(g_{j}^{\prime}\right)_{+}(t) d t
$$


где

$$
e_{j}(z)=\left\{t \in\left(t_{j}, t_{j+1}\right):\left(g_{j}^{\prime}\right)_{+}(t)>z\right\}
$$

причем $\operatorname{mes} e_{j}(z)=\xi$.

Множество $e_{j}(z)$ состоит из непересекающихся интервалов $\left(a_{k}, b_{k}\right) \subset\left(t_{j}, t_{j+1}\right)$ таких, что

$$
\left(g_{j}^{\prime}\right)_{+}\left(a_{k}\right)=\left(g_{j}^{\prime}\right)_{+}\left(b_{k}\right)=r\left(\left(g_{j}^{\prime}\right)_{+}, x\right)=z, \quad\left(g_{j}^{\prime}\right)_{+}(t)>z, \quad t \in\left(a_{k}, b_{k}\right) .
$$

Покажем, что при сделанных предположениях такой интервал единственный.

Предположим, что в множестве $e_{j}(x)$ найдется по крайней мере два непересекающихся интервала $\left(a_{1}, b_{1}\right)$ и $\left(a_{2}, b_{2}\right)$, удовлетворяющих условиям (4.18). Можно выбрать $h$ настолько малым, что при $k=1,2$ внутри $\left(a_{k}, b_{k}\right)$ существует интервал $\left(a_{k}^{\prime}, b_{k}^{\prime}\right) \subset e_{j}(z+h)$, причем, если

$$
z+h=\left(g_{j}^{\prime}\right)_{+}\left(a_{k}^{\prime}\right)=\left(g_{j}^{\prime}\right)_{+}\left(b_{k}^{\prime}\right)=r\left(\left(g_{j}^{\prime}\right)_{+}, \xi-\gamma_{0}\right),
$$

то для $\xi-\gamma_{0}<t<\xi$ будет $\delta(t)<0$. В силу последнего условия, если $r\left(\left(\widetilde{\varphi}_{j}^{\prime}\right)_{+}, \xi-\gamma\right)=$ $z+h$, то $\gamma>\gamma_{0}$. Очевидно,

$$
\gamma_{0}=b_{1}-b_{1}^{\prime}+b_{2}-b_{2}^{\prime}+a_{1}^{\prime}-a_{1}+a_{2}^{\prime}-a_{2} .
$$

Выберем на промежутке монотонности $\left[t_{j}, t_{j}+\pi / 2 n\right]$ функции $\left(\widetilde{\varphi}_{j}^{\prime}\right)_{+}$точки $\alpha$ и $\alpha^{\prime}$ таким образом, чтобы было

$$
\begin{aligned}
\left(\widetilde{\varphi}_{j}^{\prime}\right)_{+}(\alpha) & =\left(g_{j}^{\prime}\right)_{+}\left(a_{k}\right)=\left(g_{j}^{\prime}\right)_{+}\left(b_{k}\right)=z, & k & =1,2, \\
\left(\widetilde{\varphi}_{j}^{\prime}\right)_{+}\left(\alpha^{\prime}\right) & =\left(g_{j}^{\prime}\right)_{+}\left(a_{k}^{\prime}\right)=\left(g_{j}^{\prime}\right)_{+}\left(b_{k}^{\prime}\right)=z+h, & k & =1,2 .
\end{aligned}
$$

Тогда в силу предложения 5.6.6 из [1] будем иметь

$$
\left|a_{k}^{\prime}-a_{k}\right| \geqslant\left|\alpha^{\prime}-\alpha\right|, \quad\left|b_{k}-b_{k}^{\prime}\right| \geqslant\left|\alpha^{\prime}-\alpha\right|, \quad k=1,2,
$$

и, значит,

$$
\gamma_{0} \geqslant 4\left|\alpha^{\prime}-\alpha\right|=2 \gamma>\gamma,
$$

что противоречит условию $\gamma_{0}<\gamma$.

Итак, множество $e_{j}(z)$ состоит из одного интервала $(a, b)$, поэтому отрезок $[c, d] \subset$ $\left[t_{j}, t_{j+1}\right]$, который содержит этот интервал и удовлетворяет условиям

$$
\left(g_{j}^{\prime}\right)_{+}(c)=\left(g_{j}^{\prime}\right)_{+}(d)=0, \quad\left(g_{j}^{\prime}\right)_{+}(t)>0, \quad c<t<d,
$$

тоже единственный. Применив предложение 5.6.5 из [1], получим неравенство

$$
\int_{c}^{a}\left(g_{j}^{\prime}\right)_{+}(t) d t+\int_{b}^{d}\left(g_{j}^{\prime}\right)_{+}(t) d t \geqslant 2 \int_{t_{j}}^{\alpha}\left(\widetilde{\varphi}_{j}^{\prime}\right)_{+}(t) d t
$$

на основании которого, используя (4.15), можем написать

$$
\begin{aligned}
\int_{0}^{\xi} r\left(\left(\widetilde{\varphi}_{j}^{\prime}\right)_{+}, t\right) d t & =\int_{t_{j}}^{t_{j}+\pi / n}\left(\widetilde{\varphi}_{j}^{\prime}\right)_{+}(t) d t-2 \int_{t_{j}}^{\alpha}\left(\widetilde{\varphi}_{j}^{\prime}\right)_{+}(t) d t \\
& \geqslant \int_{t_{j}}^{t_{j+1}}\left(g_{j}^{\prime}\right)_{+}(t) d t-\int_{c}^{a}\left(g_{j}^{\prime}\right)_{+}(t) d t-\int_{b}^{d}\left(g_{j}^{\prime}\right)_{+}(t) d t \\
& =\int_{0}^{\xi} r\left(\left(g_{j}^{\prime}\right)_{+}, t\right) d t
\end{aligned}
$$


откуда сразу следует (4.17). Таким образом, нами доказано, что для любого $j=$ $0,1, \ldots, n-1$ имеет место (4.14).

Теперь докажем, что для любой функции $g \in W_{\infty}^{r+1}$ такой, что

$$
g\left(t_{1}\right)=g\left(t_{2}\right)=\cdots=g\left(t_{n}\right), \quad g^{\prime}\left(t_{1}\right)=g^{\prime}\left(t_{2}\right)=\cdots=g^{\prime}\left(t_{n}\right)=0,
$$

имеет место неравенство

$$
\int_{0}^{t} r\left(\left(g^{\prime}\right)_{ \pm}, u\right) d u \leqslant \int_{0}^{t} r\left(\left(\widetilde{\varphi}^{\prime}\right)_{ \pm}, u\right) d u=\int_{0}^{t} r\left(\left(\varphi_{n, r}\right)_{ \pm}, u\right) d u, \quad t \in[0,2 \pi] .
$$

Доказательство проведем для положительных частей. Случай отрицательных частей разбирается аналогично.

Пусть $t \in[0,2 \pi], j=0,1, \ldots, n-1$, обозначим

$$
\sigma_{j}(t)=\operatorname{mes}\left\{u \in\left[t_{j}, t_{j+1}\right]:\left(g_{j}^{\prime}\right)_{+}(u) \geqslant r\left(\left(g^{\prime}\right)_{+}, t\right)\right\},
$$

тогда

$$
t=\sum_{j=0}^{n-1} \sigma_{j}(t)
$$

Для $t \in[0,2 \pi]$, учитывая неравенство (4.17), можем написать

$$
\begin{aligned}
\int_{0}^{t} r\left(\left(g^{\prime}\right)_{+}, u\right) d u & =\sum_{j=0}^{n-1} \int_{0}^{\sigma_{j}(t)} r\left(\left(g_{j}^{\prime}\right)_{+}, u\right) d u \\
& \leqslant \sum_{j=0}^{n-1} \int_{0}^{\sigma_{j}(t)} r\left(\left(\widetilde{\varphi}_{j}^{\prime}\right)_{+}, u\right) d u=\sum_{j=0}^{n-1} \int_{0}^{\sigma_{j}(t)} r\left(\left(\widetilde{\varphi}_{0}^{\prime}\right)_{+}, u\right) d u .
\end{aligned}
$$

Отметим, что функция

$$
F(t)=\int_{0}^{t} r\left(\left(\widetilde{\varphi}_{0}^{\prime}\right)_{+}, u\right) d u
$$

выпукла вверх на $[0,2 \pi / n]$. Применяя неравенство Йенсена, получим

$$
\begin{aligned}
\sum_{j=0}^{n-1} \int_{0}^{\sigma_{j}(t)} r\left(\left(\widetilde{\varphi}_{0}^{\prime}\right)_{+}, u\right) d u & \leqslant n \int_{0}^{(1 / n) \sum_{j=0}^{n-1} \sigma_{j}(t)} r\left(\left(\widetilde{\varphi}_{0}^{\prime}\right)_{+}, u\right) d u \\
& =n \int_{0}^{t / n} r\left(\left(\widetilde{\varphi}_{j}^{\prime}\right)_{+}, u\right) d u=\int_{0}^{t} r\left(\left(\widetilde{\varphi}^{\prime}\right)_{+}, u\right) d u .
\end{aligned}
$$

Таким образом, неравенство (4.19) установлено, и, следовательно, (4.13) выполняется также для $m=r$.

Из (4.13) следует, что функции $g^{(m-r+1)}, m \geqslant r$, и $\varphi_{n, r}$ удовлетворяют условиям теоремы 1.3 .13 (см. [26]), из которой следует, что для всех $\lambda \in \mathbb{R}$ и $t \in[0,2 \pi]$ выполняются неравенства

$$
\int_{0}^{t} r\left(\left(g^{(m-r+1)}-\lambda\right)_{ \pm}, u\right) d u \leqslant \int_{0}^{t} r\left(\left(\varphi_{n, r}-\lambda\right)_{ \pm}, u\right) d u
$$

Используя предложение 1.3.14 из [26], можем написать

$$
\int_{0}^{2 \pi} \Pi(f, t) \Pi\left(g^{(m-r+1)}, t\right) d t \leqslant \int_{0}^{2 \pi} \Pi(f, t) \Pi\left(\varphi_{n, r}, t\right) d t .
$$


Из (4.12) и последнего неравенства сразу следует оценка сверху для $E$ :

$$
E \leqslant \sup _{\substack{f \in F \\ f \perp 1}} \int_{0}^{2 \pi} \Pi(f, t) \Pi\left(\varphi_{n, r}, t\right) d t .
$$

Оценка снизу очевидно следует из неравенства

$$
d_{2 n}\left(W^{r} F, L_{1}\right) \leqslant E\left(W^{r} F, S_{2 n, m}^{2}\right)_{1}
$$

и соотношения (2.4).

Таким образом, соотношение (3.1) доказано. Что касается соотношения (3.2), то оно следует из равенства

$$
\sup _{\substack{\|f\|_{p} \leqslant 1 \\ f \perp 1}} \int_{0}^{2 \pi} \Pi(f, t) \Pi\left(\varphi_{n, r}, t\right) d t=\left\|\varphi_{n, r}\right\|_{p^{\prime}} .
$$

Теорема доказана.

ЗАмечАниЕ 1. Неравенство (4.14), по-видимому, представляет и самостоятельный интерес. Отметим, что подобные интегральные представления для перестановок функций $f \in W_{\infty}^{r+1}$ таких, что $\|f\|_{\infty} \leqslant\left\|\varphi_{n, r+1}\right\|_{\infty}$, на отрезках длины $\pi / n$ были получены в [27; теорема 2], а на произвольных отрезках длины меньшей длины периода в [28].

ДОКАЗАТЕЛЬСТвО ТЕОРЕМЫ 2. Используя теорему двойственности для наилучшего $L_{1}$-приближения подпространством [6; теорема 1.4.1], получим

$$
E:=E\left(W^{r} H^{\omega}, S_{2 n, m}^{2}\right)_{1}=\sup _{f \in W^{r} H^{\omega}} \sup _{\substack{\|g\|_{\infty} \leqslant 1 \\ g \perp S_{2 n, m}^{2}}} \int_{0}^{2 \pi} f(t) g(t) d t .
$$

После $r$-кратного интегрирования по частям с использованием леммы 1 можем написать

$$
E=\sup _{\substack{f \in H^{\omega} \\ f \perp 1}} \sup _{\substack{g \in W_{\infty}^{r} \\ g^{(r)} \perp S_{2 n, m}^{2}}} \int_{0}^{2 \pi} f(t) g(t) d t=\sup _{\substack{f \in H^{\omega} \\ f \perp 1}} \sup _{\substack{g \in W_{\infty}^{m+1} \\ g\left(t_{1}\right)=\cdots=g\left(t_{n}\right) \\ g^{\prime}\left(t_{1}\right)=\cdots=g^{\prime}\left(t_{n}\right)=0}} \int_{0}^{2 \pi} f(t) g^{(m-r+1)}(t) d t .
$$

Через $R(f, t)$ будем обозначать через $\Sigma$ перестановку $2 \pi$-периодической функции $f$ на промежутке длины $2 \pi$ [6; с. 294].

Из (4.21), применяя теорему 7.5.1 из [1], получим

$$
E \leqslant \sup _{\substack{g \in W_{\infty}^{m+1} \\ g\left(t_{1}\right)=\cdots=g\left(t_{n}\right) \\ g^{\prime}\left(t_{1}\right)=\cdots=g^{\prime}\left(t_{n}\right)=0}} \int_{0}^{2 \pi} R\left(g^{(m-r)}, t\right) \omega^{\prime}(t) d t .
$$

Оценим правую часть (4.22). Пусть сначала $m \geqslant r+2, g \in W_{\infty}^{m+1}$ и

$$
g\left(t_{1}\right)=g_{t_{2}}=\cdots=g\left(t_{n}\right), \quad g^{\prime}\left(t_{1}\right)=g^{\prime}\left(t_{2}\right)=\cdots=g^{\prime}\left(t_{n}\right)=0 .
$$

Тогда функция $g$ удовлетворяет условиям теоремы 3 , в силу которой

$$
\left\|g^{(m-r)}\right\|_{\infty} \leqslant\left\|\varphi_{n, r+1}\right\|_{\infty}
$$


и, следовательно,

$$
\max _{a, b \in[0,2 \pi]}\left|\int_{a}^{b} g^{(m-r)}(t) d t\right| \leqslant 2\left\|g^{(m-r-1)}\right\|_{\infty} \leqslant 2\left\|\varphi_{n, r+2}\right\|_{\infty} .
$$

Значит, для такой функции $g$ выполняются условия теоремы 6.8 .5 из [1], в силу которой

$$
\int_{0}^{2 \pi} R\left(g^{(m-r)}, t\right) \omega^{\prime}(t) d t \leqslant \int_{0}^{2 \pi} R\left(\varphi_{n, r+1}, t\right) \omega^{\prime}(t) d t .
$$

Пусть теперь $m=r+1$. В этом случае неравенство (4.24) мы установим непосредственно.

Так как $g \in W_{\infty}^{r+2}$ и удовлетворяет условию (4.23), то по теореме $3\left\|g^{\prime}\right\|_{\infty} \leqslant$ $\left\|\varphi_{n, r+1}\right\|_{\infty}$. Теперь в силу теоремы 6.8 .4 из [1] заключаем, что для любого $x \in$ $(0, \pi / n)$ выполняется по крайней мере одно из неравенств

$$
\left|R^{\prime}\left(g^{\prime}, x\right)\right| \leqslant\left|R^{\prime}\left(\varphi_{n, r+1}, x\right)\right|
$$

или

$$
R\left(g^{\prime}, x\right) \leqslant R\left(\varphi_{n, r+1}, x\right) .
$$

Это означает, что разность $\delta(x)=R\left(\varphi_{n, r+1}, x\right)-R\left(g^{\prime}, x\right)$ на промежутке $[0,2 \pi]$ либо неотрицательна либо меняет знак с “+” на “-” в единственной точке $t_{0} \in[0,2 \pi]$. Отметим, что в случае, когда $\delta(x) \geqslant 0$ для всех $t_{0} \in[0,2 \pi]$, выполнение неравенства (4.24) очевидно. Рассмотрим случай, когда $\delta(x)$ меняет знак на $[0,2 \pi]$. Из полученного при доказательстве теоремы 1 неравенства (4.19) при $t=2 \pi$ следует, что

$$
\int_{0}^{2 \pi} r\left(g_{ \pm}^{\prime}, u\right) d u \leqslant \int_{0}^{2 \pi} r\left(\left(\varphi_{n, r+1}\right)_{ \pm}, u\right) d u
$$

откуда

$$
\int_{0}^{2 \pi} r\left(g^{\prime}, u\right) d u \leqslant \int_{0}^{2 \pi} r\left(\left(\varphi_{n, r+1}\right), u\right) d u .
$$

Учитывая тот факт [1; с. 131, 141], что для $\psi \in L_{1}$

$$
\|\psi\|_{1}=\int_{0}^{2 \pi} r(\psi, u) d u=\int_{0}^{2 \pi} R(\psi, u) d u,
$$

на основании (4.27) можем утверждать, что $\int_{0}^{2 \pi} \delta(t) d t \geqslant 0$. Рассуждая далее, как при доказательстве теоремы 6.7.6 из [1], имеем (ниже через $\omega_{+}^{\prime}(t)$ обозначена правая производная функции $\omega(t)$, которая в силу выпуклости вверх функции $\omega(t)$ существует в каждой точке)

$$
\begin{aligned}
\int_{0}^{2 \pi} & \omega^{\prime}(t) R\left(\varphi_{n, r+1}, t\right) d t-\int_{0}^{2 \pi} \omega^{\prime}(t) R\left(g^{\prime}, t\right) d t \\
& =\int_{0}^{2 \pi} \omega^{\prime}(t) \delta(t) d t=\int_{0}^{t_{0}} \omega^{\prime}(t) \delta(t) d t+\int_{t_{0}}^{2 \pi} \omega^{\prime}(t) \delta(t) d t \\
& \geqslant \omega^{\prime}\left(t_{0}\right) \int_{0}^{t_{0}} \delta(t) d t+\omega^{\prime}\left(t_{0}\right) \int_{t_{0}}^{2 \pi} \delta(t) d t=\omega^{\prime}\left(t_{0}\right) \int_{0}^{2 \pi} \delta(t) d t \geqslant 0 .
\end{aligned}
$$


Справедливость (4.24) установлена и при $m=r+1$. Учитывая (4.22), (4.24), а также равенство [6; с. 299]

$$
\left\|f_{n, m}(\omega ; \cdot)\right\|_{1}=\int_{0}^{2 \pi} R\left(\varphi_{n, m+1}, t\right) \omega^{\prime}(t) d t
$$

получаем оценку сверху для величин $E\left(W^{r} H^{\omega}, S_{2 n, m}^{2}\right)_{1}$ :

$$
E\left(W^{r} H^{\omega}, S_{2 n, m}^{2}\right)_{1}=E \leqslant \int_{0}^{2 \pi} R\left(\varphi_{n, r+1}, t\right) \omega^{\prime}(t) d t=\left\|f_{n, r}(\omega ; \cdot)\right\|_{1} .
$$

Оценка снизу следует из неравенства

$$
E\left(W^{r} H^{\omega}, S_{2 n, r}^{2}\right)_{1} \geqslant d_{2 n}\left(W^{r} H^{\omega}, L_{1}\right)
$$

и $(2.3)$.

Теорема доказана.

\section{СПИСОК ЦИТИРОВАННОЙ ЛИТЕРАТУРЫ}

[1] Н. П. Корнейчук, Экстремальные задачи теории приближения, Наука, М., 1976.

[2] Н. П. Корнейчук, А. А. Лигун, В. Г. Доронин, Аппроксимация с ограничениями, Наукова думка, Киев, 1982.

[3] М. А. Красносельский, Я. Б. Рутицкий, Выпуклые функции и пространства Орлича, Современные проблемы математики, Физматгиз, М., 1958.

[4] С. Г. Крейн, Ю.И. Петунин, Е. М. Семенов, Интерполяиия линейных операторов, Наука, М., 1978.

[5] Х. Трибель, Теория интерполяции, функциональные пространства, дифференциальнъе операторы, Мир, М., 1980.

[6] Н. П. Корнейчук, Точнье константы в теории приближения, Наука, М., 1987.

[7] С.М. Никольский, "Приближение функций тригонометрическими полиномами в среднем", Изв. АН СССР. Сер. матем., 10:3 (1946), 207-256.

[8] Л.В. Тайков, "О приближении в среднем некоторых классов аналитических функций”, Приближение функиий в среднем, Тр. МИАН, 88, Наука, М., 1967, 61-70.

[9] С. П. Туровец, "О наилучшем приближении в среднем дифференцируемых функций”, Докл. АН УССР. Сер. А, 5 (1968), 417-421.

[10] A. A. Ligun, "Inequalities for upper bounds of functions", Anal. Math., 2:1 (1976), 11-40.

[11] Ю.И. Маковоз, "Поперечники некоторых функциональных классов в пространстве L", Изв. АН БССР. Сер. физ.-матем. наук, 1969, № 4, 19-28.

[12] Ю.Н. Субботин, "Поперечник класса $W^{r} L$ в $L(0,2 \pi)$ и приближение сплайн-функциями", Матем. заметки, 7:1 (1970), 43-52.

[13] Ю.Н.Субботин, "Приближение сплайн-функциями и оценки поперечников", Приближение периодических функций, Тр. МИАН, 109, Наука, М., 1971, 35-60.

[14] Ю. И. Маковоз, "Об одном приеме оценки снизу поперечников множеств в банаховых пространствах", Матем. сб., 87:1 (1972), 136-142.

[15] А. А. Лигун, "О поперечниках некоторых классов дифференцируемых периодических функций”, Матем. заметки, 27:1 (1980), 61-75.

[16] Ю. И. Маковоз, "Поперечники соболевских классов и сплайны, наименее уклоняющиеся от нуля", Матем. заметки, 26:5 (1979), 805-812.

[17] A. Pinkus, "On n-widths of periodic functions", J. Analyse Math., 35 (1979), 209-235.

[18] V.F. Babenko, "Approximations, widths and optimal quadrature formulae for classes of periodic functions with rearrangement invariant sets of derivatives", Anal. Math., 13:4 (1987), 281-306. 
[19] Н. П. Корнейчук, "Верхние грани наилучших приближений на классах дифференцируемых периодических функций в метриках $C$ и $L "$, Докл. AH CCCP, 190 (1970), 269-271.

[20] Н. П. Корнейчук, "Экстремальные значения функционалов и наилучшее приближение на классах периодических функций”, Изв. АН СССР. Сер. матем., 35:1 (1971), 93-124.

[21] Н.П. Корнейчук, "Наилучшее приближение сплайнами на классах периодических функций в метрике $L "$, Матем. заметки, 20:5 (1976), 655-664.

[22] В.П. Моторный, В.И.Рубан, "Поперечники некоторых классов дифференцируемых периодических функций в пространстве L", Матем. заметки, 17:4 (1975), 531-543.

[23] В. И. Рубан, "Поперечники множеств в пространствах периодических функций", Докл. АН CCCP, 225:1 (1980), 34-35.

[24] А.Н. Колмогоров, “О неравенствах между верхними гранями последовательных производных функций на бесконечном интервале”, Ученые записки Моск. гос. ун-та. Матем., 30:3 (1939), 3-16.

[25] С.М. Никольский, "Приближение функций тригонометрическими полиномами в среднем", Изв. АН СССР. Сер. матем., 10:3 (1946), 207-256.

[26] Н. П. Корнейчук, В. Ф. Бабенко, А. А. Лигун, Экстремальные свойства полиномов и сплайнов, Наукова думка, Киев, 1992.

[27] В. И. Иванов, "Приближение функций из $C^{r}$ сплайнами минимального дефекта", $M a$ тем. заметки, 43:6 (1988), 746-756.

[28] А. В. Московский, "Некоторые экстремальные свойства дифференцируемых периодических функций, полиномов и сплайнов на отрезках меньших периода", Изв. ТулГУ. Сер. Матем. Мех. Информ., 2:1 (1996), 169-175.

\section{В. $\Phi$. Бабенко}

Днепропетровский национальный университет,

г. Днепропетровск,

Институт прикладной математики и механики НАН Украины,

г. Донецк

E-mail: babenko.vladislav@gmail.com

\section{Н. В. Парфинович}

Днепропетровский национальный университет

E-mail: nparfinovich@mail.ru
Поступило 07.03.2008 